\title{
GPPS-CH-2020-0027
}

\section{EXPERIMENTAL INVESTIGATION OF BLEED AIR EXTRACTION THROUGH A CIRCUMFERENTIAL GAP IN A LOW SPEED AXIAL COMPRESSOR STAGE}

\author{
Melanie Achmus, Jens Friedrichs \\ Institute of Jet Propulsion and Turbomachinery \\ Technische Universität Braunschweig \\ m.achmus@ifas.tu-braunschweig.de \\ Braunschweig, Germany
}

\author{
Bernd Becker \\ Rolls-Royce Deutschland Ltd. and Co. KG \\ bernd.becker@rolls-royce.com \\ Blankenfelde-Mahlow, Germany
}

\begin{abstract}
For the supply of secondary air systems such as turbine cooling, oil sump and cabin ventilation, bleed air is taken from the compressor of aircraft engines. The design objective is to extract the required fluid with as little losses as possible. Bleed air is usually extracted at the casing downstream of the stator row. By shifting the extraction position by half a stage upstream, an improvement of the compressor efficiency could be achieved. In this measuring campaign the effect of a bleed air geometry is experimentially investigated downstream of the stator blade row as well as downstream of the rotor blade row of a 1.5-stage axial compressor. In the studies presented here, the differences of the configurations with respect to stage aerodynamics and aerodynamics of the bleed air geometry are shown. The aim is to investigate the potential of bleed air extraction downstream of the rotor blade row. Since the investigation of bleed air extraction in the rotating test rig has a higher complexity compared to the pure axial compressor, the following will not only deal with the results of the measurement campaign. In addition, some general considerations and lessons learned are discussed in relation to the bleed air removal research.
\end{abstract}

\section{INTRODUCTION}

Bleed air extraction plays an important role when it comes to the efficiency and stability of the axial compressor of aircraft engines and stationary gas turbines. Therefore an efficient bleed air system should meet different criteria. When extracting fluid for seconday air systems, the smallest possible influence on core flow of the compressor is desirable. Also the extracted fluid itself should experience minimum losses. The higher the static pressure recovery of the extraction port, the higher the pressure available for secondary air systems in the plenum. An aerodynamic improvement of static pressure recovery of the extraction ports would allow a shifting of the the extraction position to the upstream stages, this would have a positive impact on the specific fuel consumption.

In the past, several studies have been presented on this topic, which dealt with the geometry and positioning of the bleed air removal port. Poenick [1] and Leishman [2],[3] investigated bleed air removal through circular holes. Zimmermann [4], Leishman [2], Peltier [5], [6], Gomes [7], [8], Grimshaw [9], [10] and Aufderheide [11] examined circumferential bleed ports. So far most research was done in non-rotating systems or in cascades. In the test section presented in the following, the aerodynamic behaviour of the bleed port geometry and the interaction with the 1.5-stage axial compressor is examined in a rotating system. In addition, previous investigations have mainly dealt with the bleed air extraction downstream of a row of stator blades. This is all too understandable, since downstream of the rotor blade row there are comparatively high speeds due to the swirl in the flow. At high extraction mass flows, there is therefore a justified concern that the bleed air duct will be blocked. Nevertheless, first fundamental investigations of the influence of the extraction position downstream of the rotor blade row are carried out here, which should lead to a better understanding of the effects on the stage aerodynamics. If, in future research projects, it were possible to convert much of the twist behind the rotor into static pressure, the extraction position downstream of the rotor blade row would have great potential.

\section{NOMENCLATURE}

$\begin{array}{ll}c_{a x} & \text { Axial velocity } \\ c_{p} & \text { isobaric heat capacity } \\ \Delta H & \text { Enthalpy difference } \\ h_{r e l} & \text { Relative spanwise position } \\ \mathrm{M} & \text { Torque }\end{array}$

$\left(\frac{m}{s}\right)$

$\left(\frac{J}{k g K}\right)$

$(J)$

$(N m)$ 


$\begin{array}{lll}\mathrm{n} & \text { Rotational speed } & (\mathrm{Hz}) \\ \dot{m} & \text { Mass flow } & \left(\frac{\mathrm{kg}}{\mathrm{s}}\right) \\ \overline{\dot{m}} & \text { Average mass flow } & \left(\frac{\mathrm{kg}}{\mathrm{s}}\right) \\ P & \text { Power } & (\mathrm{W}) \\ p & \text { Static pressure } & (\mathrm{Pa}) \\ \bar{p} & \text { Average static pressure } & (\mathrm{Pa}) \\ p_{t} & \text { Total pressure } & (\mathrm{Pa}) \\ \bar{p}_{t} & \text { Average total pressure } & (\mathrm{Pa}) \\ q & \text { Dynamic pressure } & (\mathrm{Pa}) \\ R & \text { Universal gas constant } & \left(\frac{\mathrm{J}}{\mathrm{kgK}}\right) \\ T & \text { Static Temperature } & (\mathrm{K}) \\ T_{t} & \text { Total Temperature } & (\mathrm{K}) \\ \bar{T} & \text { Average total Temperature } & (\mathrm{K}) \\ V & \text { Volume } & \left(\mathrm{m}^{3}\right) \\ Y & \text { Work } & (\mathrm{Nm}) \\ \beta & \text { Turning angle } & \left({ }^{\circ}\right) \\ \kappa & \text { Isentropic exponent } & \\ \eta & \text { Efficiency } & \\ \pi & \text { Pressure ratio } & \\ \zeta & \text { Pressure loss coefficient (absolute) } \\ \zeta & \text { Pressure loss coefficient (relative) }\end{array}$

\section{Abbreviations}

BdR Bleed air removal downstream of Rotor row

BdS Bleed air removal downstream of Stator row

BMF Bleed air mass flow

DP Design point

HPC High pressure compressor

IGV Inlet guide vanes

LSRC Low speed research compressor

ME Measuring plane in test section

SPR Static pressure recovery

VSV Variable stator vanes

\section{Subscripts}

$t$ loss

sh

$s$

1

2

3

15

25

Caused by mechanical losses

Shaft

Static

Total value

$\mathrm{ME1}$, inlet rotor row

ME2, inlet stator row

ME3, stage exit

ME15, upstream of bleed port inlet (BdR)

ME25, upstream of bleed port inlet (BdS)

\section{EXPERIMENTAL SETUP}

The investigations presented in this paper were conducted at the Low Speed Research Compressor (LSRC) of TU Braunschweig which was build for the experimental examination of up to 2.5 axial compressor stages. In order to allow the investiagation of bleed air removal a new test section and a bleed air removal system was build. For more details regarding the bleed air removal system and the LSRC please refer to [12]. The test section contains a 1.5-stage axial compressor which is representative for an HPC-stage. The stage is designed as a repeating stage, where the IGV generates the neccessary pre swirl. The compressor contains $45 \mathrm{IGV}$ vanes, 45 shrouded stator vanes and 43 rotor blades. With a casing diameter of $600 \mathrm{~mm}$ and a hub diameter

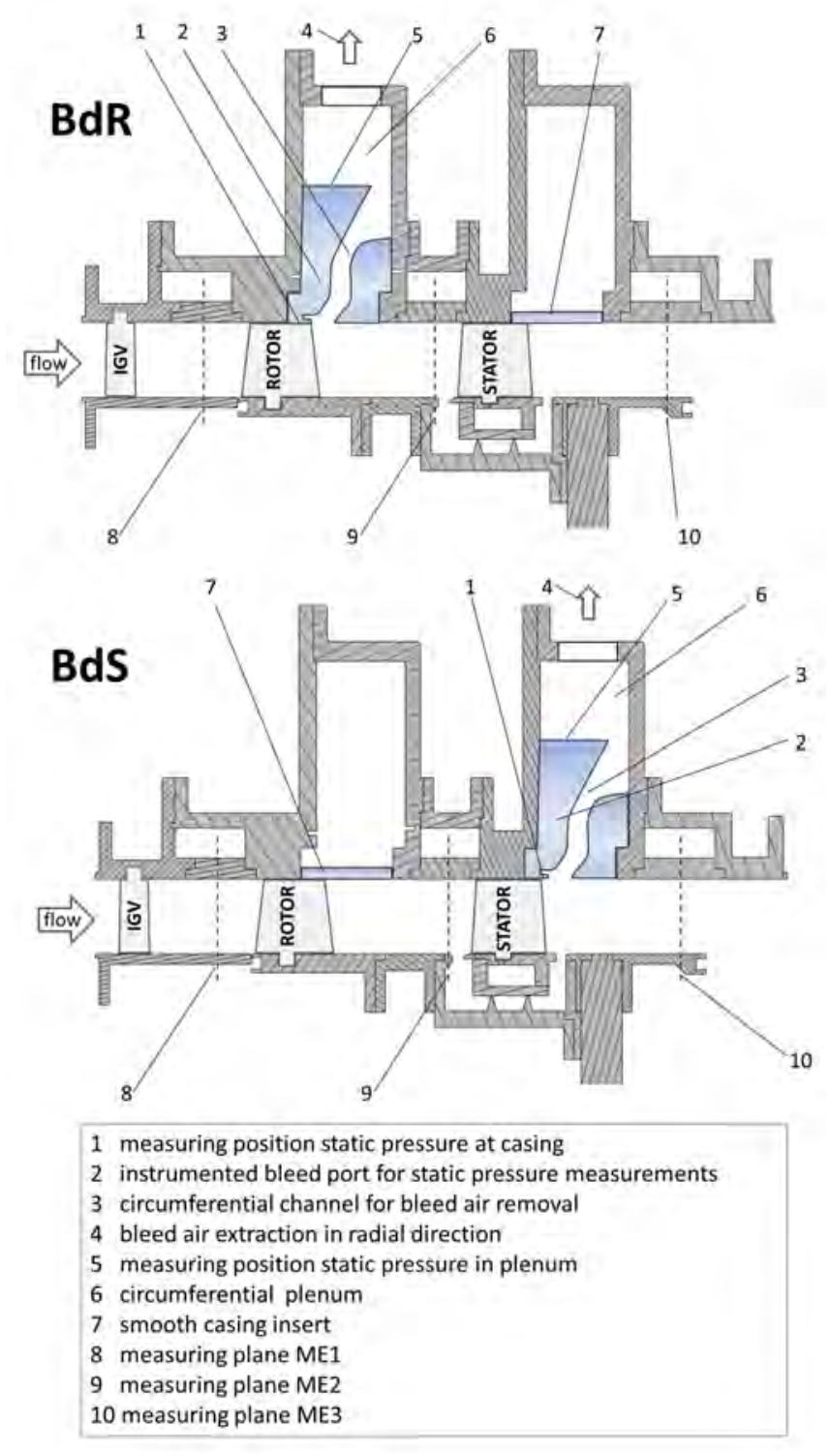

FIGURE 1. Examined compressor stage configurations for bleed air removal downstream of the rotor row $(\mathrm{BdR})$ and bleed air removal downstream of the stator row (BdS)

of $450 \mathrm{~mm}$ the hub to tip ratio is 0.75 . The seal gap height of the stator shroud is $3 \%$ of duct height and the rotor tip clearance is $0.67 \%$ of duct height. As the stator is constructed as VSV row, the resulting hub and tip clearances are quite high at design stagger angle and vary along the chord. At the hub the maximum clearance is $1 \mathrm{~mm}$, at the casing it is up to $1.7 \mathrm{~mm}$. As the aim of this study is not the investigation of the absolute stage performance but a comparison between two different bleed air locations, a resulting deterioration in operational performance is acceptable.

The two investigated configurations are shown in figure 1 . The configuration at the top shows bleed air removal downstream of the rotor row $(\mathrm{BdR})$ and the configuration at the bottom shows bleed air removal downstream of the stator row (BdS). In some diagrams these configurations will in the following be compared with the reference configuration with smooth casing. The test 
section contains a circumferential plenum downstream of the rotor row and a second plenum downstream of the stator row (6). The plenum chambers are integrated in the casing which has the advantage that no interfering struts for casing stability are needed in the area of the bleed port. This way arbitrary bleed air geometries can be installed in the plenum chamber. With a smooth casing insert (7) the plenum which is not needed is closed. In the reference configuration (smooth casing) both plenums are closed. The applied bleed port geometry forms a circumferential gap at the casing with an axial width of 0.3 times the duct height. The extracted fluid passes the bleed air duct (3) and then enters the plenum (6). It is extracted through 8 extraction pipes evenly spaced around the circumference. In this measuring campaign the same amount of bleed air is removed through each pipe, which implies a uniform bleed air removal.

As figure 1 shows, the axial distances between the rows are wider than in a real compressor stage. This is necessary for the installations of the five-hole probes. When looking at the results it must be kept in mind that the axial distances are not realistic as a certain mixing of the wake takes place before reaching the measuring plane and the downstream row.

The experiments were conducted at a constant aerodynamic rotor speed of $2800 \mathrm{rpm}$ and the data analysis is limited to the design operating point $\left(\dot{m}_{1, D P}=\right.$ constant $)$. For each of the two configurations different amounts of bleed mass flow (BMF) from $0 \%$ to $14.3 \%$ of the inlet mass flow were conducted.

\section{METHODOLOGY}

The presented results are based on wake-flow measurements with pneumatic five-hole probes, which were conducted in the measuring planes ME1 (8), ME2 (9) and ME3 (10), compare figure 1. A segment of $8^{\circ}$ was measured in each plane, which corresponds to a stator passage. Each segment comprises 650 measuring points. As the bleed air is drawn evenly around the circumference, it can be assumed that one segment is representative of the entire circumference. To determine the stage efficiency, the drive torque of the rotor shaft and the temperatures in ME1 and ME3 were also measured. In order to be able to get information about the course of the static pressure in the extraction duct, a $45^{\circ}$ segment of the upstream component was instrumented with holes for static wall pressure measurements (2). The lowest hole is located on the casing (1), the upper hole is located inside the plenum (5). In total, the segment is provided with 120 holes, 10 each in the same radial position. A circumferential averaging allows the visualization of a representative pressure curve and the calculation of the static pressure recovery (SPR) of the extraction duct.

\section{Calculation of efficiency and stage pressure ratio}

In the measurements of stage characteristics carried out at the LSRC in the past, in which no fluid was extracted or blown in, the stage efficiency could be determined in good approximation on the basis of static values. The efficiency could be determined from the static wall pressures, the temperatures and the torque of the drive shaft. This made it possible to map the complete characteristic curve of a compressor configuration with comparatively little effort. Since the stages investigated are repeating low speed stages, it could be assumed that the dynamic pressure in ME1 corresponds to the dynamic pressure in ME3. Although this is an LSRC-specific efficiency definition, it has been shown to be very close to the performance based on total values for pressure and temperature. However, if, as in the measurement campaign presented here, fluid is extracted between the two measurement levels, the simplifying assumptions made in the past are no longer valid.

The dynamic pressure downstream of the stage no longer corresponds to the dynamic pressure upstream of the stage. Since up to $15 \%$ of the mass flow is extracted between the measuring levels, there is a correspondingly lower mass flow downstream of the stage. This leads to a rise in static pressure in ME3 when increasing the BMF. Both for the efficiency and for the pressure ratio in the case of bleed air extraction, it is therefore not possible to display these values on the basis of static values. For the calculation of the flow parameters based on total values, the wake-flow measurements are used. As these measurements are very time-consuming, the data are only available at certain operating points. This is why the following evaluation will be limited to the analysis of the design point of the stage. The following applies to the stage efficiency and pressure ratio, the explanation of the terminology used can be found in figure 2 .

Two different mean total pressures can be calculated for each measuring plane located upstream of the sampling position. In ME1 for example $\bar{p}_{t 1, \dot{m}_{1}}$ denotes the total pressure averaged over the entire duct height, while $\bar{p}_{t 1, \dot{m}_{3}}$ stands for the average total pressure of the mass flow later passing ME3. The stage pressure ratio can therefore be characterised in two different ways.

$$
\pi_{31, \dot{m}_{1}}=\frac{\bar{p}_{t 3}}{\bar{p}_{t 1, \dot{m}_{1}}} \quad \text { and } \quad \pi_{31, \dot{m}_{3}}=\frac{\bar{p}_{t 3}}{\bar{p}_{t 1, \dot{m}_{3}}}
$$

In both equations $\bar{p}_{t 3}$ is the total pressure of the fluid in ME3. $\pi_{31, \dot{m}_{1}}$ describes the pressure ratio when the entire cross-section of the duct is considered in ME1 and $\pi_{31, m_{3}}$ describes the pressure ratio which the fluid $\dot{m}_{3}$ has experienced. $\dot{m}_{1}$ is the mass flow that enters the stage and $\dot{m}_{3}$ is the mass flow that remains in the compressor after bleed air extraction. Since the total pressure level near the casing is lower than in the middle of the duct due to the boundary layer influence, $\pi_{31, \dot{m}_{3}}$ is lower than $\pi_{31, \dot{m}_{1}}$.

The efficiency of the compressor stage is the ratio of the energy increase which the fluid experiences when flowing through the stage and the work expended by the compressor rotor for achieving this. For the calculation of the power $P$ applied for the energy increase, the share of the torque $M$ is of interest, which was actually spent for conveying the mass flow $\dot{m}_{1}$. The torque $M_{\text {loss }}$, which is needed to compensate the friction in the bearings for example, is therefore subtracted from the measured torque $M_{\text {shaft }}$ in the efficiency calculation. The following applies

$$
M=M_{\text {shaft }}-M_{\text {loss }}
$$

and

$$
P=2 \pi n M \quad \text {. }
$$



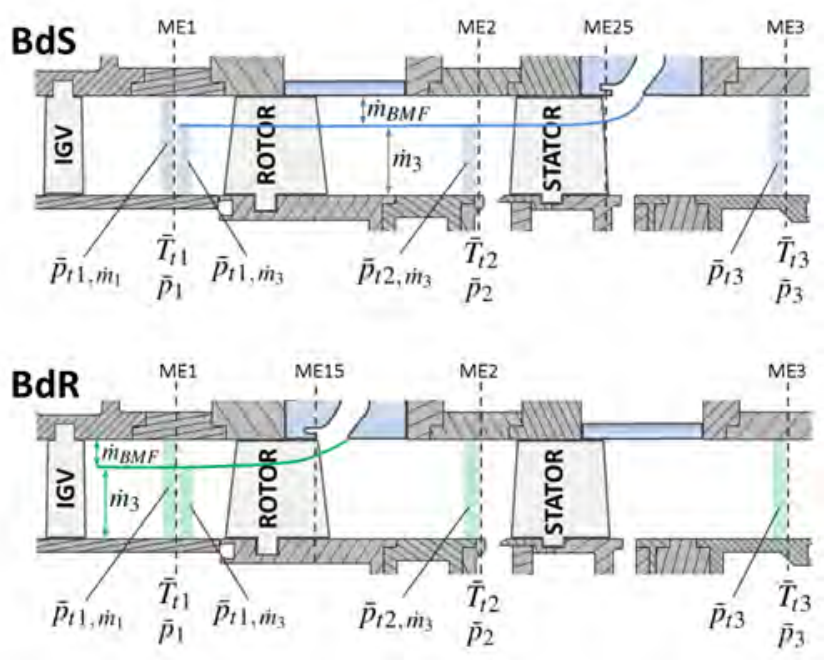

FIGURE 2. Schematic illustration of the flow distribution in the compressor stage, presentation of used terminology

The increase in the energy of a fluid can be described by the total enthalpy difference $d H$, assuming an ideal gas and an isentropic change of state. For the change of state of a mass flow $\dot{m}$ from a state 1 to a state 3 the enthalpy difference is

$$
d H=\dot{m} c_{p} d T=\dot{m} c_{p}\left(T_{t 3}-T_{t 1}\right)
$$

The specific isobaric heat capacity $c_{p}$ can be expressed by the isentropic exponent $\kappa$ and the specific gas constant $R$. By using the isentropic relationship, the total enthalpy difference can be represented based on the pressures and temperatures of the fluid. For the efficiency of a compressor stage located between the two measuring levels 1 and 3 generally the following applies

$$
\eta_{31}=\frac{\dot{m} c_{p}\left(T_{t 3}-T_{t 1}\right)}{P}=\frac{\dot{m}\left[\frac{\kappa R}{\kappa-1} T_{t 1}\left(\left(\frac{p_{t 3}}{p_{t 1}}\right)^{\frac{\kappa-1}{\kappa}}-1\right)\right]}{P} .
$$

In this case the enthalpy of $\dot{m}$ was increased by $d H_{31}$ by applying the power $P$. If a part of the mass flow is extracted between the levels, this must be taken into account when calculating the efficiency. In the configurations examined here, the work recorded via the torque was performed on the entire fluid $\dot{m}_{1}$. In ME3, however, only $\dot{m}_{3}$ remains after the removal of $\dot{m}_{B M F}$. The efficiency of the overall process in BdS results in

$$
\begin{aligned}
& \eta_{31}=\underbrace{\frac{\left(\dot{m}_{3} c_{p}\left(T_{t 3}-T_{t 1}\right)\right)}{P}}_{\eta_{31, \dot{m}_{3}}}+\underbrace{\frac{\left(\dot{m}_{B M F} c_{p}\left(T_{t 25}-T_{t 1}\right)\right)}{P}}_{\eta_{31, B M F}}, \\
& \eta_{31, \dot{m}_{3}}=\frac{\dot{m}_{3}\left[\frac{\kappa R}{\kappa-1} T_{t 1}\left(\left(\frac{\bar{p}_{t 3}}{\bar{p}_{t 1, \dot{m}_{3}}}\right)^{\frac{\kappa-1}{\kappa}}-1\right)\right]}{P},
\end{aligned}
$$

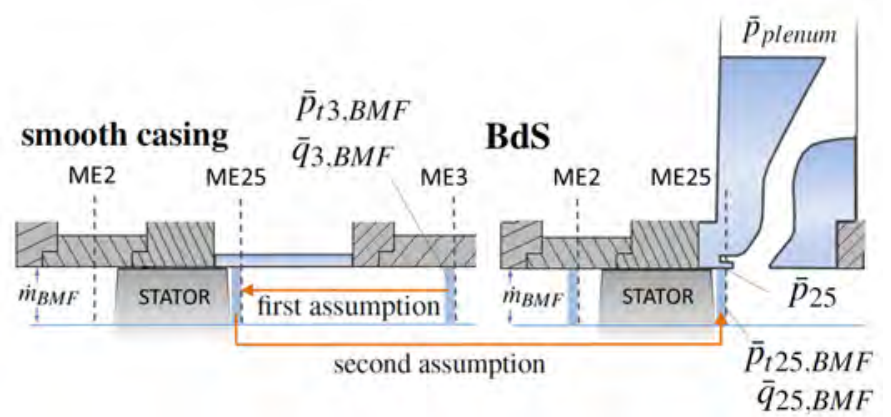

FIGURE 3. illustration of simplifying assumptions made in order to calculate the efficiency $\eta_{31, B M F}$

$$
\eta_{31, B M F}=\frac{\dot{m}_{B M F}\left[\frac{\kappa R}{\kappa-1} T_{t 1}\left(\left(\frac{\bar{p}_{t 25, B M F}}{\bar{p}_{t 1, B M F}}\right)^{\frac{\kappa-1}{\kappa}}-1\right)\right]}{P}
$$

Where $T_{t, 25}$ represents the total temperature of the bleed air mass flow shortly before it is extracted. In the case of BdR the temperature $T_{t, 15}$ must be used (compare figure 2).

The first term of the equation indicates how effectively the energy level of the mass flow remaining in the compressor was increased. The second term describes the enthalpie increase of the bleed air mass flow until the time of extraction. For the compressor itself $\eta_{31, \dot{m}_{3}}$ is the more decisive factor. It gives an indication of how effectively the work expended has been converted into the energy increase of the mass flow $\dot{m}_{3}$ which stays in the compressor. All values required for the calculation of $\eta_{31, \dot{m}_{3}}$ are available as measurement data (see figure 2). Due to limited accessibility for probes in the measuring section the efficiency of the energy increase of $\dot{m}_{B M F}$ can only be calculated as an approximation based on simplifying assumptions. The simplifications made are explained below using the example of BdS using figure 3. It is assumed that in the smooth casing configuration the flow condition in ME3 corresponds to the flow condition in ME25. Losses due to friction are therefore neglected at this point (assumption 1). It is also assumed that the flow condition in ME25 in the smooth casing configuration corresponds to the flow condition in ME25 in BdS (assumption 2). Furthermore, it is assumed that the work required to increase the energy of $\dot{m}_{B M F}$ is the same in the smooth casing configuration and $\mathrm{BdS} / \mathrm{BdR}$. As will be reported later $P$ actually differs in the different configurations. For the calculation of $\eta_{31, B M F}$ this difference is neglected as the proportion of $\dot{m}_{B M F}$ in $\dot{m}_{1}$ is small.

\section{Calculation of static pressure recovery}

The static pressure recovery SPR provides an information about the effectiveness of the conversion of kinetic energy into potential energy of a bleed port geometry. In the case of BdS it is defined as

$$
S P R=\frac{\bar{p}_{\text {plenum }}-\bar{p}_{25}}{\bar{q}_{25, B M F}},
$$

where $\bar{p}_{\text {plenum }}$ is the static pressure in the plenum and $\bar{p}_{25}$ is the static pressure at the casing. The dynamic pressure $\bar{q}_{25, B M F}$ is 


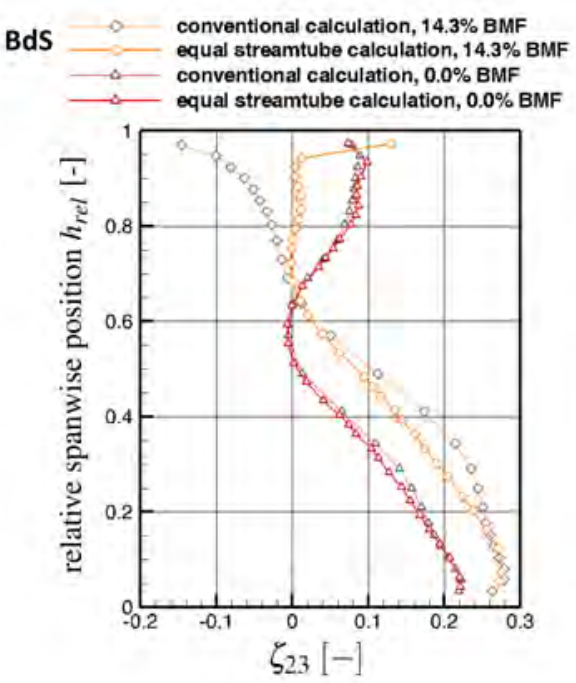

FIGURE 4. Comparison of the two different ways of measurement data processing using the example of $\zeta_{23}$, DP

a quantity which, analogous to the static pressure $\bar{p}_{t 25, B M F}$, was calculated using the two simplifying assumptions presented in figure 3. For the calculation of SPR in BdR the values in ME15 must be used instead of ME25. Another possibility of comparing the bleed duct performance is the static pressure ratio

$$
\pi_{s, B M F}=\frac{\bar{p}_{\text {plenum }}}{\bar{p}_{25}}
$$

\section{Calculation of flow coefficients on same stream tube}

The stage performance is analysed using the flow turning $\Delta \beta$ and the pressure loss coefficient $\zeta$. It applies:

$\zeta_{12}^{\prime}=\frac{\bar{p}_{t 1, \dot{m}_{3}}^{\prime}-\bar{p}_{t 2, \dot{m}_{3}}^{\prime}}{\bar{p}_{t 1, \dot{m}_{3}}^{\prime}-\bar{p}_{1}}$

$$
\zeta_{23}=\frac{\bar{p}_{t 2, \dot{m}_{3}}-\bar{p}_{t 3}}{\bar{p}_{t 2, \dot{m}_{3}}-\bar{p}_{2}} .
$$

Where $\zeta_{23}$ is the loss coefficient in the absolute frame of reference and $\zeta_{12}^{\prime}$ is the loss coefficient in the relative frame of reference. These characteristic values are determined on the basis of the wake-flow measurement data in the three measuring planes. If the same grid is selected for the measuring positions of the probe in each measuring plane, the values at the same duct height can be used after circumferential averaging the data, since there is no significant shift of the streamlines with the hub and casing radius present in the LSRC. In the case of bleed air extraction, as will be shown below, there is a significant local deflection of the streamlines, so that in this case using data from the same relative spanwise position is not expedient. For this reason, a routine was developed which uses the measured values at the duct heights up to which the same cumulative mass flow was conveyed. In this way a stream tube from ME1 can also be found in ME3. Figure 4 shows the pressure loss coefficient $\zeta_{23}$ for $0 \% \mathrm{BMF}$ and $14.3 \%$ $\mathrm{BMF}$ for the conventional calculation and the equal stream tube calculation as an example. The course along the relative spanwise position is plottet, where $h_{r e l}=0$ corresponds to the hub
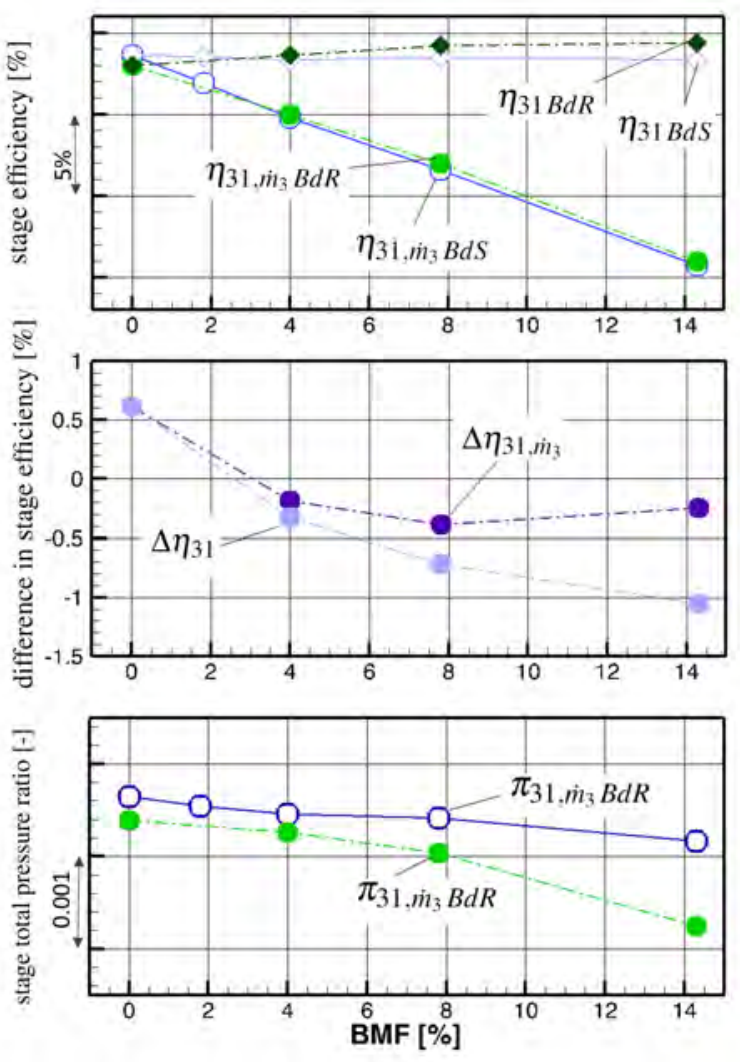

FIGURE 5. Stage efficiencies $\eta_{31}$, and $\eta_{31, \dot{m}_{3}}$, difference in stage efficiency $\Delta \eta=\eta_{B d S}-\eta_{B d R}$, stage total pressure ratio of fluid remaining in the compressor $\pi_{31, \dot{m}_{3}}$

and $h_{\text {rel }}=1$ corresponds to the casing. It can be seen that there are hardly any differences between the two types of calculating the coefficient in the case of no bleed air extraction; the assumption of identical flow tubes at the same duct height is valid in this case. The more bleed air is extracted the greater the resulting differences. In the case of $14.3 \% \mathrm{BMF}$, the pressure loss in the lower half of the duct is overestimated, while it is severely underestimated on the casing side. Here, using data from the same spanwise position even results in a large range of physically illogical negative loss coefficients.

\section{RESULTS AND DISCUSSION Efficiency and Pressure Rise}

Figure 5 (above) shows the course of the stage efficiency at the design point with different $\mathrm{BMF}$ for $\mathrm{BdR}$ and $\mathrm{BdS}$. In this figure the overall stage efficiency $\eta_{31}$ is shown as well as the efficiency of the energy increase of the stage exit mass flow $\eta_{31, \dot{m}_{3}}$. The difference between the two courses represents $\eta_{31, B M F}$ as can be seen from equation 7 . With increasing BMF $\eta_{31, \dot{m}_{3}}$ decreases nearly linearly. $\eta_{31}$ on the other hand remains with larger values. This is not surprising at first, since a lower outlet mass flow leads to a lower gain in absolute internal energy of the fluid. The more fluid is extracted the more energy put into the system is lost. Interesting, however, is the difference in efficiency between BdS and BdR which is additionally shown in figure 5 (middle). 

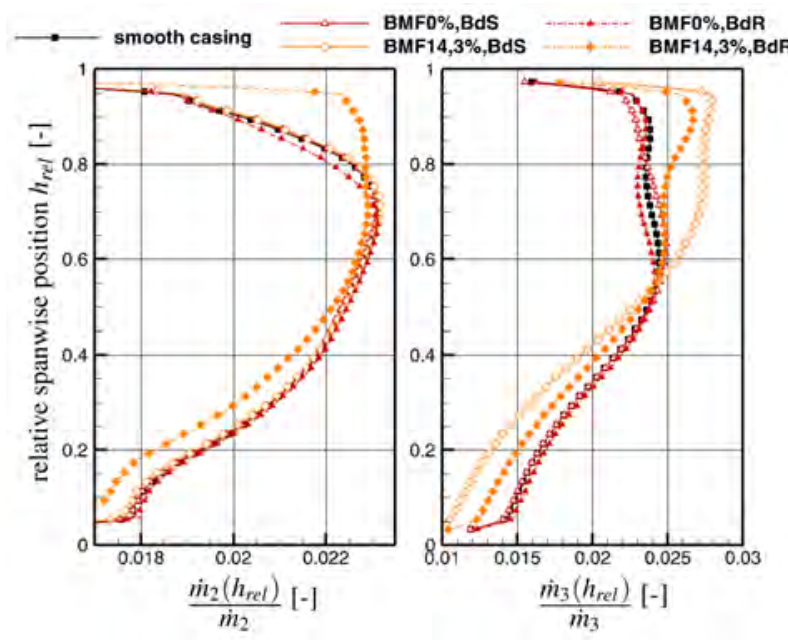

FIGURE 6. Flow redistribution caused by bleed air removal of $0 \%$ and $14.3 \%$ BMF for BdR and BdS in ME2 and ME3

It can be seen that at $0 \%$ extraction (cavity open but no bleed air extraction) BdS has a slightly higher efficiency than BdR. This means that the mere existence of the circumferential gap downstream of the rotor leads to higher losses. From about 3\% BMF this trend changes and $\mathrm{BdR}$ shows higher efficiencies.

In the case of BdR $\eta_{31}$ even increases with increasing BMF while in the case of BdS $\eta_{31}$ is on a relatively constant level. In BdS the same flow conditions are present in the stage until the point of extraction. BdS thus does not cause upstream effects which have perceptable impact on the stage efficiency $\eta_{31}$. The effects of the bleed air removal in this configuration would become more apparent in a second stage downstream of the investigated stage. $\mathrm{BdR}$ in contrast influences $\eta_{31}$ in a positive way. However, it should be noted that the total pressure rise achieved by BdR is lower, compare figure 5 (bottom). The phenomenon of a higher efficiency in BdR in comparison to BdS is assumed to have two reasons.

- In the configuration $\mathrm{BdR}$ the torque of the shaft that is needed to deliver the stage mass flow decreases with increasing BMF. In BdS the power $\mathrm{P}$ stays at a constant level when the BMF is increased. In BdR P decreases whith increasing BMF. This is due to the reduced back pressure in ME2. When extracting bleed air in the configuration BdR less work is needed to drive the shaft at a constant rotating speed and stage inlet mass flow.

- In BdR $\dot{m}_{B M F}$ does not experience total pressure losses in the stator row. So the energy level of the extracted fluid is higher in the case of BdR. That is also the reason why $\Delta \eta_{31}$ has higher absolute values than $\Delta \eta_{31, \dot{m}_{3}}$

\section{Analysis of wake flow measurements}

The flow redistribution resulting from bleed air removal for the two configurations is shown in figure 6 . To get a clearer presentation, only the data for $0 \%$ and $14.3 \%$ BMF are shown here. In order to achieve comparability of the mass flow distributions despite the different total mass flow rates for different BMF, the

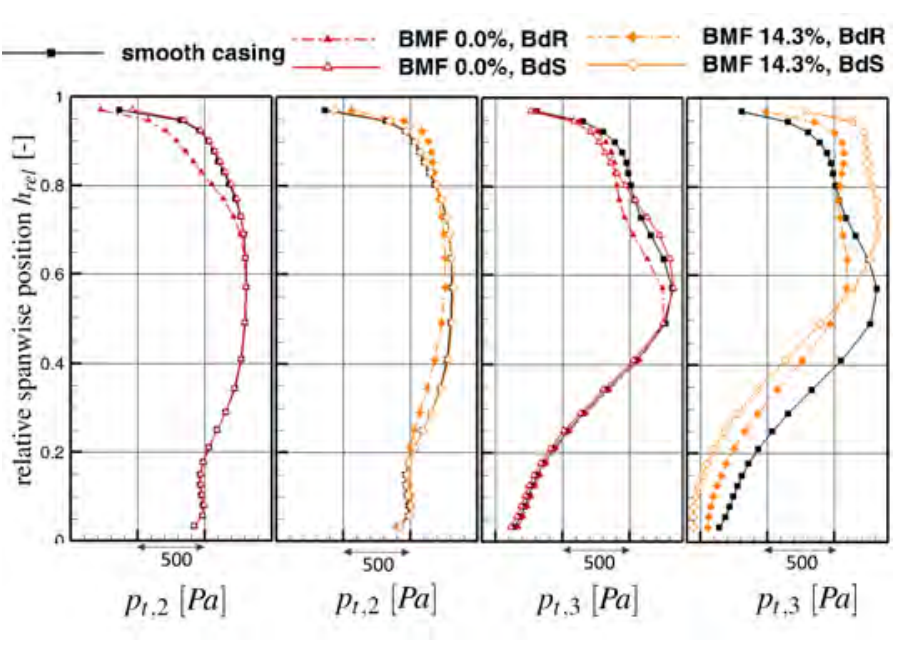

FIGURE 7. Radial course of total pressure $p_{t, 2}$ and $p_{t, 3}$ caused by bleed air removal of $0 \%$ and 14.3\% BMF for BdR and BdS in ME2 and ME3

curves were divided by the corresponding cumulative total mass flow.

ME2: There is no difference between smooth casing and $0 \%$ $\mathrm{BMF}$ for BdS. Even at $14.3 \% \mathrm{BMF}$ there is no upstream effect. In the case of BdR, $0 \% \mathrm{BMF}$ results in a lower mass flow in the upper $30 \%$ of the duct. This is due to the blockage effect of the gap downstream of the rotor row. Due to turbulence caused by fluid flowing in and out of the gap, there is a loss of momentum near the casing and thus a lower mass flow. At 14.3\% BMF BdS leads to a strong flow redistribution. This is due to the fact that lossy fluid with low kinetic energy at casing is extracted and fluid with high kinetic energy is shifted from the middle of the duct towards the casing. So the upper $20 \%$ of the duct shows a strongly increased mass flow rate. In the remaining part of the duct, this flow redistribution leads to a lower mass flow rate. The resulting difference in velocity has an effect on the flow to the stator vanes. The change of the flow parameters will later be illustrated in the form of velocity triangles.

ME3: The comparison of the two configurations shows that $\mathrm{BdS}$ causes a stronger flow redistribution both at $0 \% \mathrm{BMF}$ and at $14.3 \%$ BMF. For a potentially downstream rotor, BdR therefore means a comparatively smaller deviation from the design configuration with regard to mass flow distribution.

The total pressure curves, which correlate strongly with the mass flow distribution, are shown in figure 7.

0\% BMF: A comparison of $p_{t 2, B d R}$ and $p_{t 3 . B d s}$ shows that the mere existence of the circumferential gap has a stronger impact in the case of BdR. This explains the lower efficiency at $0 \%$ BMF.

14,3\% BMF: Due to the extraction of lossy fluid and the resulting flow redistribution, the areas of high total pressure are located closer to the casing. The higher the BMF, the higher the total pressure in the area close to the casing. This total pressure increase is paid for with a total pressure loss at the hub, which is due to a lower mass flow and thus lower velocities. This effect can be observed for both BdR in ME2 and BdS in ME3. In ME3 $\mathrm{BdR}$ shows a lower total pressure level than BdS. For this reason $\pi_{31, \dot{m}_{1}}(\mathrm{BdS})$ is higer than $\pi_{31, \dot{m}_{1}}(\mathrm{BdR})$. Nevertheless, the shape 

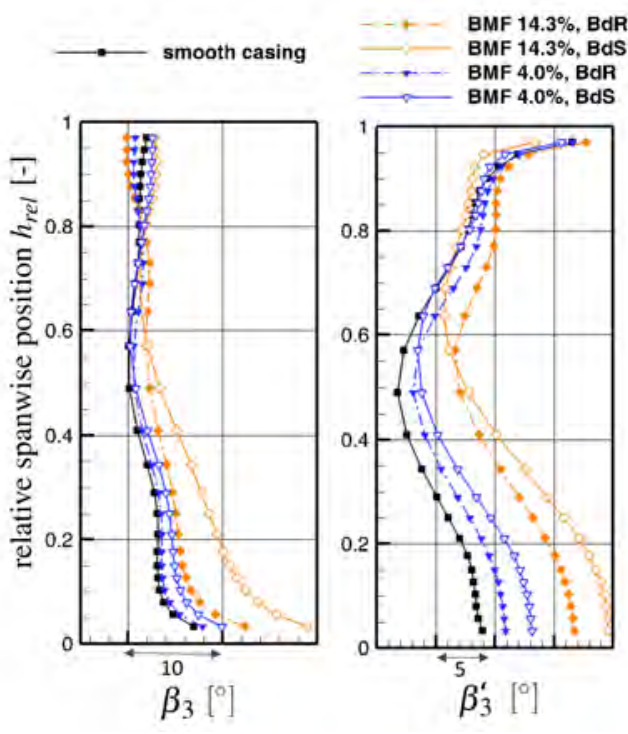

FIGURE 8. Radial course of flow angle $\beta_{3}$ and $\beta_{3}^{\prime}$ caused by bleed air removal for BdR and BdS in ME3

of the pressure profile of BdR as a whole - analogoue to the mass flow distribution - is closer to the pressure profiles shape of the design configuration (smooth casing).

The fact that BdR shows a smaller deviation from the design configuration than BdS becomes also clear when looking at the flow angles $\beta_{3}$ and $\beta_{3}^{\prime}$ in figure 8. Especially in the lower half of the duct, BdS leads to higher flow angles in the absolute frame of reference as well as in the relative frame of reference. For the rotor of a potentially downstream stage, this means a comparatively steeper inflow in this area and thus a greater risk of flow separation. This trend is reversed in the area close to the casing, where BdR leads to a steeper flow towards the following rotor.

The velocity triangles in figure 9 generated from the wake-flow data illustrate the differences in the flow conditions between $\mathrm{BdR}$ and BdS. Exemplarily the case of $14.3 \% \mathrm{BMF}$ is shown. Looking at ME2 it can be seen that at the casing BdR leads to a less inclined flow towards the stator leading edge, while at the hub a flow with higher incidence angles occurs. Downstream of the stator, in ME3, there is therefore a reduced deflection at the hub which results in a steeper flow towards a potentially downstream rotor. However, BdS nevertheless leads to a greater reduction in deflection in this area. The velocity triangles provide a good illustration of how the change in flow angle is related to the change in axial velocity. How the rotor of a second stage reacts to the changed flow conditions caused by $\mathrm{BdR}$ or $\mathrm{BdS}$ depends strongly on its profile design. If the hub area is comparatively sensitive to a deviation in the flow angle, $\mathrm{BdR}$ is of advantage. If the rotor blade in the casing area reacts sensitively to deviations, BdS is advantageous. This means that whether BdR or BdS has a stronger influence on the compressor characteristics can only be assessed in combination with the downstream stage.

On the basis of the velocity triangles in figure 9, it has already been shown that in the case of $\mathrm{BdS}$ the extraction of bleed air leads to very low speeds in the hub area. Another good

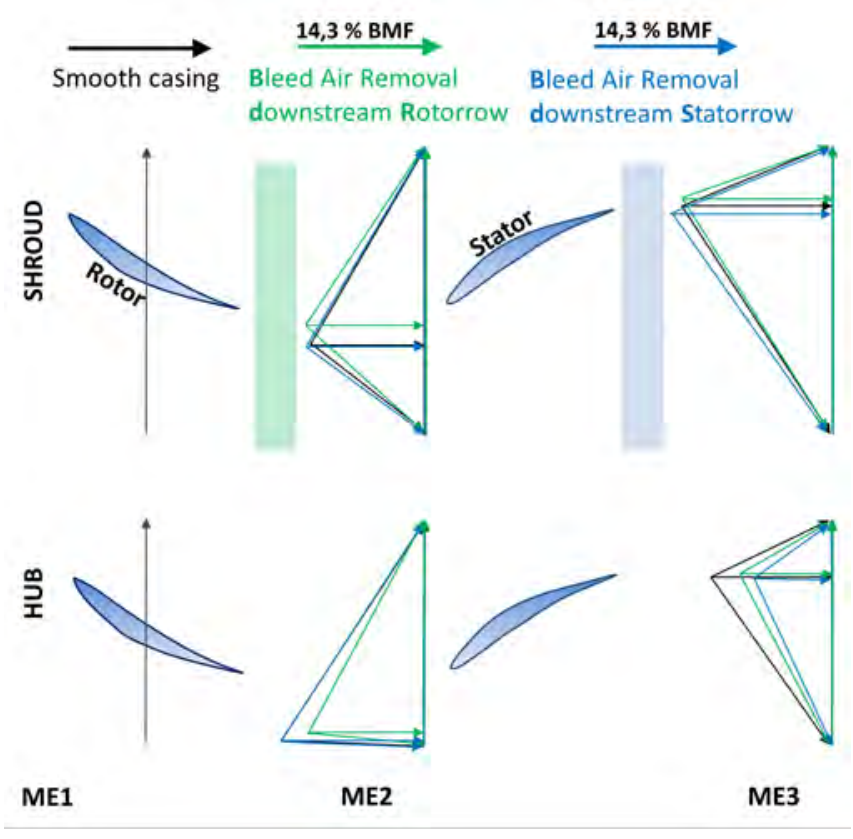

FIGURE 9. Velocity triangles for smooth casing and $14.3 \% \mathrm{BMF}$ at hub and casing for BdR and BdS

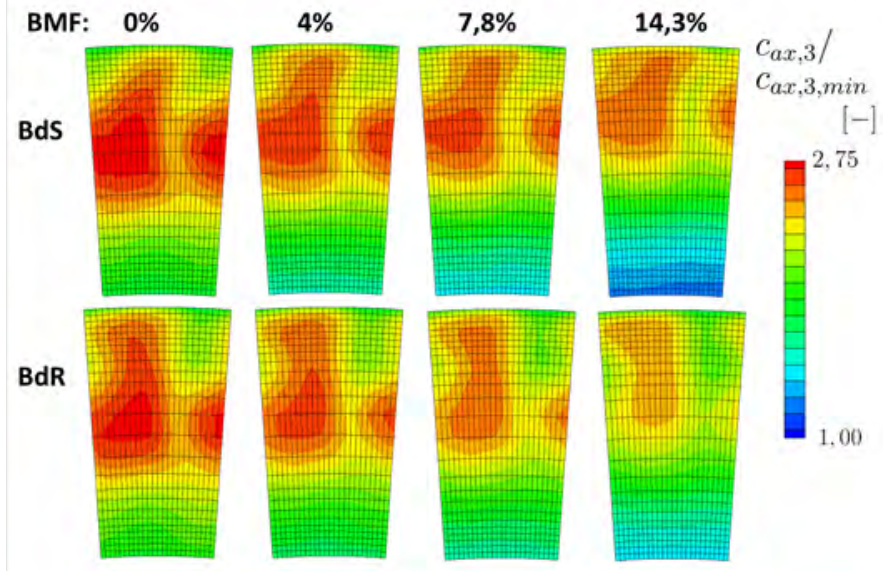

FIGURE 10. Axial velocity contour in stator passage at stage exit for different BMF in ME3 for BdR and BdS

impression of the influence of bleed air extraction is given by the illustration of the wake-flow data of a blade passage, as can be seen in figure 10. Shown here is the axial velocity in ME3 in DP for different BMF for BdR and BdS. In both configurations there is a shift of the areas with high kinetic energy towards the casing. This leads to a lower velocity with increasing BMF and thus lower mass flow rates in the hub area. In general, it can be said that BdS leads to a stronger non-uniformity in radial direction.

Figure 11 (left) shows the radial course of the loss coefficients between the measurement planes ME1 and ME2 $\left(\zeta_{12}^{\prime}\right)$ and ME2 and ME3 $\left(\zeta_{23}\right)$. As described above, the calculation was carried out on the same stream tubes between the measurement planes. Since the calculation area sometimes includes the extraction position and sometimes does not, the terms rotor loss coefficient 


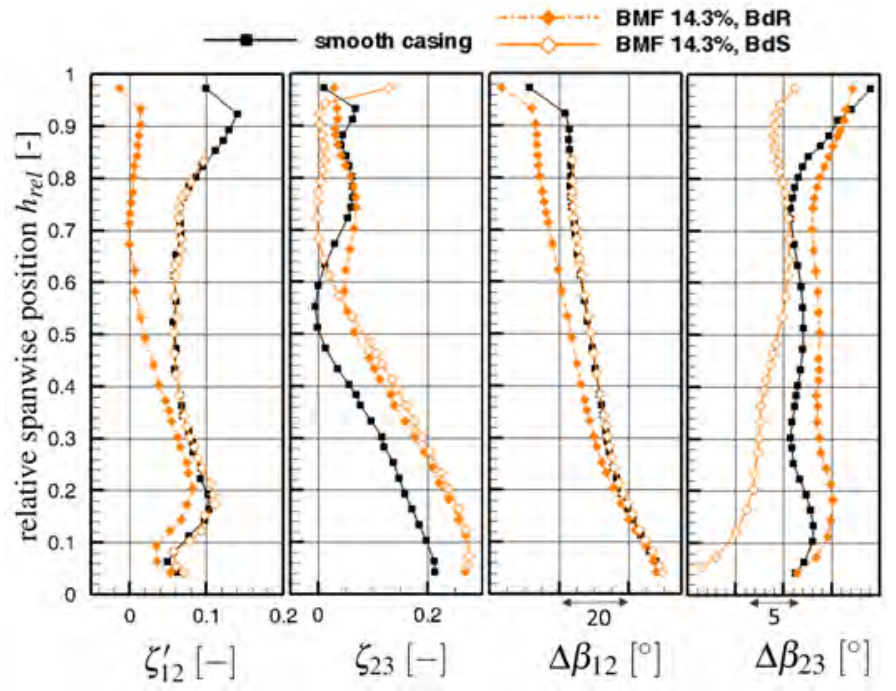

FIGURE 11. Pressure loss coefficients $\zeta_{12}^{\prime}$ and $\zeta_{23}$ and flow deflection $\Delta \beta_{12}$ and $\Delta \beta_{23}$ for the smooth casing configuration and $14.3 \% \mathrm{BMF}$ for BdR and BdS

$\zeta_{R}$ and stator loss coefficient $\zeta_{S}$ were deliberately omitted here. $\zeta_{12}^{\prime}$ : It can be seen that BdR leads to lower losses between ME1 and ME2. This is not surprising, since loss-affected fluid was extracted in the casing area. As expected, the loss coefficient is highest near the casing, but the influence of the bleed air extraction extends to the hub. In the case of $\mathrm{BdS}$ there is no significant effect on the loss coefficient $\zeta_{12, B d S}^{\prime}$. Since only the percentage of the fluid that subsequently passes through ME3 was taken into account for the calculation, the representation of the radial course only goes up to $84 \%$ of the duct height. The fluid located in the upper $16 \%$ of the duct is therefore extracted downstream of the stator.

$\zeta_{23}$ : In the casing area the pressure loss coefficient for $\mathrm{BdS}$ is lower than in the smooth casing configuration. This is again due to the extraction of loss-affected fluid. The losses between ME2 and ME3 caused by BdR are comparatively high. This can also be seen in figure 12 .

Figure 12 shows $\zeta_{12}^{\prime}$ and $\zeta_{23}$ as a function of the BMF. With increasing BMF, BdR has a positive influence on the loss coefficient $\zeta_{12}^{\prime}$ but a negative effect on $\zeta_{23}$. BdS, on the other hand, shows a more moderate change in losses with the BMF. When comparing the values, however, it must be taken into consideration that, for example, in the case of BdR between ME2 and ME3 no bleed air was taken and the lossy areas on the casing are included in the calculation. The resulting loss coefficient is correspondingly higher. I some cases bleed air is extracted between the measuring planes $\left(\zeta_{12}^{\prime}(\mathrm{BdR}), \zeta_{23}(\mathrm{BdS})\right)$ and in other cases there is no bleed air extraction between the measuring planes $\left(\zeta_{12}^{\prime}(\mathrm{BdS}), \zeta_{23}(\mathrm{BdR})\right)$. Care should therefore be taken when comparing the values as they are not pure rotor or stator coefficients.

The different losses, which occur at BdR and BdS between the measuring planes, suggest why $\mathrm{BdR}$ provides a better efficiency but a lower total pressure ratio. The loss coefficient $\zeta_{12}^{\prime}$, which decreases with increasing BMF, is responsible for the improved efficiency. As can be seen from equations 3 and
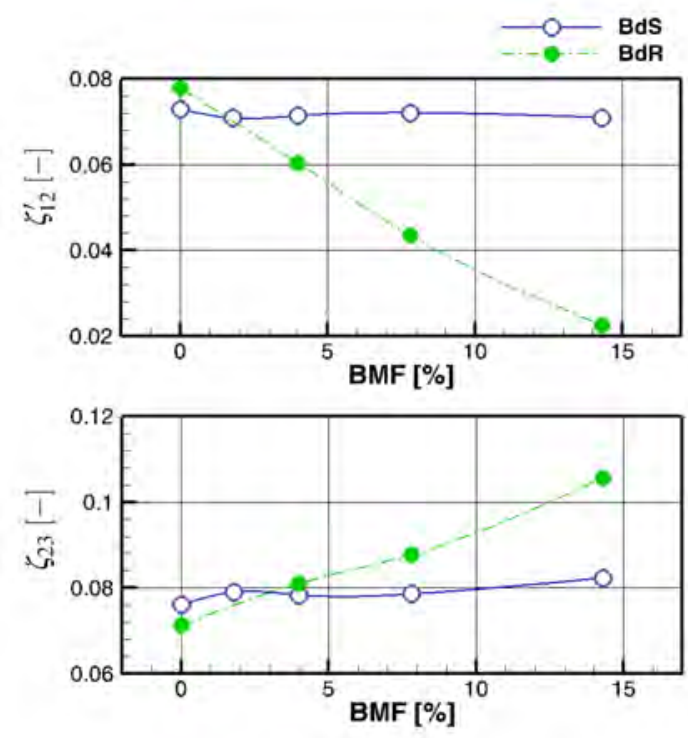

FIGURE 12. Pressure loss coefficients $\zeta_{12}^{\prime}$ and $\zeta_{23}$ as a function of $\mathrm{BMF}$ for BdR and BdS, DP

4, the denominator of the equation is dependent on the power of the drive shaft, which quantifies the work that has actually been transferred to the fluid. The less pressure losses are generated in the rotor stage, the less work has to be done to convey the same mass flow and efficiency increases. The loss coefficient $\zeta_{23}$, on the other hand, which decreases with increasing BMF, is responsible for the nevertheless lower total pressure ratio.

Figure 11 (right) shows the change in angular momentum between the measuring planes. BdR leads to a reduced deflection between ME1 and ME2. Since the values in ME1 are independent of the BMF, this is equivalent to a lower flow angle in the relative frame of reference.

For $\Delta \beta_{23, B d S}$ there is a lower deflection than in the reference case of the smooth casing configuration, while $\Delta \beta_{23, B d R}$ is higher over almost the entire duct height. When interpreting the results, however, it should be noted that (analogous to the loss coefficients) in the case of $\Delta \beta_{12, B d R}$ and $\Delta \beta_{23, B d S}$ the bleed air extraction is located in the calculation area, whereas $\Delta \beta_{12, B d S}$ and $\Delta \beta_{23, B d R}$ are actually pure blade parameters. For design reasons, it is not possible to measure the flow parameters between the trailing edge of the blade and the extraction gap. The velocity triangles that would be measured, for example, directly downstream of the rotor, are different from the velocity triangles measured downstream of the extraction position behind the rotor. Behind the extraction position there is a lower mass flow and thus on average a lower axial velocity and a higher static pressure. This causes a change in flow angles. $\Delta \beta_{12, B d R}$ and $\Delta \beta_{23, B d S}$ thus describe a change in deflection which is not alone due to the angular momentum realised by the blades.

\section{Pressure recovery in the bleed duct}

In the following the course of the static pressure distribution in the extraction port and the pressure recovery behaviour will be discussed. Figure 13 shows the static pressure at the bleed duct 


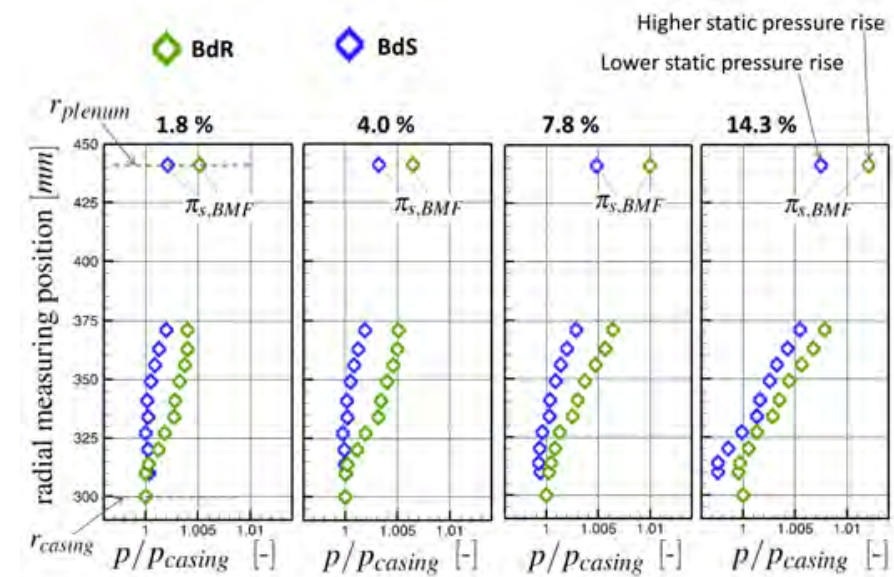

FIGURE 13. Pressure recovery in extraction duct for different BMF for BdR and BdS

wall for BdR and BdS at different BMF. The lowest measuring point is located directly downstream of the trailing edge of the blade on the casing $\left(r_{\text {casing }}\right)$, so still in the aero-path. The highest measuring point is in the plenum $\left(r_{\text {plenum }}\right)$. It is to be noted that the static pressure at the casing $\bar{p}_{25}$ is higher at $\mathrm{BdS}$ than at $\operatorname{BdR}\left(\bar{p}_{25}>\bar{p}_{15}>\right)$. In order to be able to compare the curves directly with each other, the values were related to the static pressure present at $r_{\text {casing. }}$. Additionally this plot allows conclusions to be drawn about the static pressure ratio $\pi_{s, B M F}$ in the extraction geometry, see figure 13. Besides, the difference of both pressures $\bar{p}_{\text {plenum }}-\bar{p}_{25}$, shows qualitatively the same course.

The course of the static pressure at the duct wall gives an indication of the deceleration of the flow. An increase in static pressure implies a decrease in total flow velocity. At low BMF a relatively lower deceleration is realized with $\mathrm{BdS}$. In the lower half of the extraction duct the static pressure is at a nearly constant level. It is presumed that there is a detachment that leads to reduced effective diffusion compared to the geometry area increase. With $\mathrm{BdR}$ a pressure recovery is achieved even at small BMF. When the BMF increases, the static pressure courses of BdR and BdS become closer.

As figure 13 shows, BdR realises a consistently higher $\pi_{s, B M F}$. Due to the high velocities downstream of the rotor row it applies $\bar{q}_{15}>\bar{q}_{25}$. That is why the static pressure recovery SPR of the two configurations are closer together although $\pi_{s, B M F}$ differs, as shown in figure 14 . For BMF of up to $10 \%$ it can be seen that the same geometry provides a better SPR with BdR than with BdS. For larger BMF this trend is reversed. For both configurations, the general trend is for SPR to increase with increasing BMF. At low BMF flow separations form on the duct wall. When the extracted mass flow is increased the flow reattaches and is decelerated by the widening cross-section of the duct. It is assumed that the higher SPR at low BMF achieved in BdR is related to the swirl and the velocity of the flow. The high angular momentum and the longer flowpath lead to reduced diffusion. A schematic illustration of this approach is shown in figure 15. Due to the swirl in the flow it experiences a more moderate expansion of the cross-section. In addition the higher kinetic energy of the fluid means that the flow is less prone to detachment. A

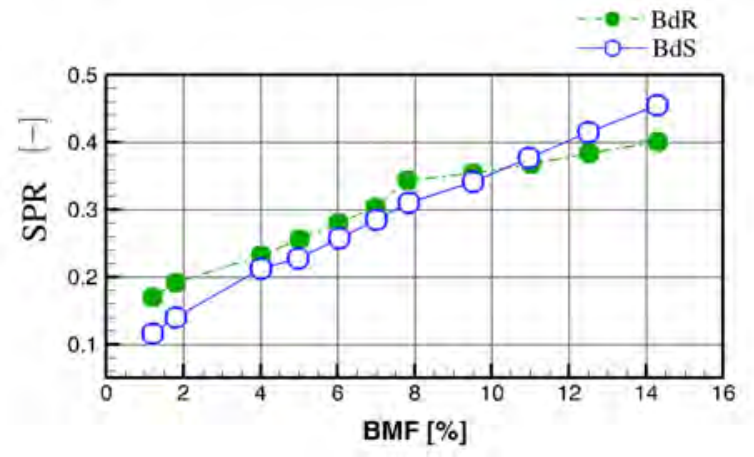

FIGURE 14. Static pressure recovery SPR achieved in BdS and BdR
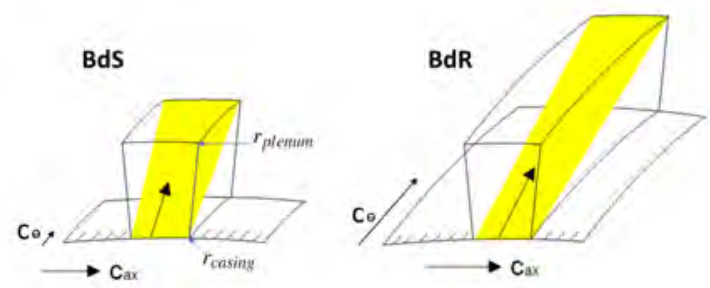

FIGURE 15. Visualisation of different diffusor effects in the same extraction port for the two configurations BdS (left) and BdR (right)

precise explanation for the deterioration of the SPR for larger BMF in the case of BdR has yet to be found. The fact that higher velocities cause higher friction losses could be one reason.

Despite the higher SPR at low BMF the static pressure in the plenum is higher in $\mathrm{BdS}$ than in $\mathrm{BdR}$. This is because in $\mathrm{BdS}$ the static pressure of the extracted fluid is higher already. In order to achieve the same pressure level within the plenum, BdR would have to be implemented in further downstream compressor stages. However, this is not desirable with regard to compressor aerodynamics. Shifting the position of bleed air extraction by half a stage upstream, an improvement of the system efficiency would be possible. However, the static pressure in the plenum at BdR must be just as high as in the case of BdS. To achieve this, a further increase in pressure recovery is necessary.

\section{CONCLUSION}

The experimental investigation of a compressor stage with additional bleed air extraction entails increased complexity. The evaluation of stage characteristics on the basis of static values (as done in previous investigations without bleed air removal) is no longer possible. Due to the flow redistribution caused by bleed air extraction, it is also necessary to calculate the aerodynamic coefficients such as $\zeta$ and $\Delta \beta$ on the same stream tube.

The evaluation of the results focused on a comparison of the aerodynamic impact of $\mathrm{BdR}$ and $\mathrm{BdS}$ on the compressor stage. The data analysis was limited to the design operating point of the stage. It was shown that 
- The existence of the circumferential gap ( $0 \%$ BMF) causes higher losses when located downstream of the rotor row than when located downstream of the stator row.

- When extracting bleed air BdR leads to a lower total pressure ratio but to a higher stage efficiency compared to BdS.

- At the stage exit BdR shows a lower impact on the flow parameters than BdS. This effect was demonstrated using the radial course of $\dot{m}_{3}, p_{t 3}, \beta_{3}$ and $\beta_{3}^{\prime}$. The comparison of the axial velocity contour at different BMF for BdR and $\mathrm{BdS}$ showed that BdS results in a higher inhomogeneity of the stage exit flow.

- In the casing area BdS leads to higher positve incidence angles for a potential downstream rotor row than BdR, in the hub area BdR leads to higher positve incidence angles for a potential downstream rotor row than BdS. The resulting effect on the compressor characteristics depend on the design of the downstream stage and can only be assesed in combination with a multi-stage configuration.

- For BMF of up to $10 \%$ the static pressure recovery SPR of the bleed air duct is higher at the installation position downstream of the rotor row. Still the static pressure in the plenum is lower.

From the authors' point of view there are two ways in which bleed air removal downstream of rotor rows can become a realistic alternative to bleed air removal downstream of stator rows. First of all, BdR must be examined in a multi-stage configuration. Should the aerodynamic influence on the stage aerodynamics prove to be advantageous, as indicated in the 1.5-stage configuration, BdR would be an additional option of extracting bleed air. If it was possible to realise a further transformation of kinetic energy into static pressure in the upper part of the extraction duct, at plenum could be achieved same (or even higher) static pressure than at $\mathrm{BdS}$.

The bleed air geometry used for this investigation was designed for BdS. The development of an extraction port designed for BdR would probably enhance the pressure recovery.

\section{ACKNOWLEDGEMENTS}

This work was funded by Rolls-Royce Deutschland and the Bundesministerium für Wirtschaft und Technologie (BMWI) according to the resultion of the German Federal Parliament under the Grant No.03ET7071N and 03ET7071I. The authors gratefully acknowledge Rolls-Royce Deutschland for their support and the permission to publish this paper.

\section{References}

[1] Poenick S., Kozulovic D., Radespiel R., Becker B., Guemmer V., 2013 "Experimental and Numerical Investigations of a Compressor Cascade Flow with Secondary Air Removal", ASME Journal of Turbomachinery Vol. 135, 021030 pp 110.

[2] Leishman B.A., Cumpsty N.A., Denton J.D., 2007 "Effect of Inlet Ramp Surfaces on the Aeodynamic Behaviour of Bleed Hole and Blee Slot Off-Take Configurations", Journal of Turbomachinery Vol. 129, p 659.

[3] Leishman B.A., Cumpsty N.A., Denton J.D., 2007 “Effect of Bleed Rate and Endwall Location on the Aerodynamic Behaviour of a Circular Hole Bleed Off-Take", Journal of Turbomachinery Vol. 129, pp 645-658.

[4] Zimmermann H. 1990 "Some Aerodynamic Aspects of Engine Secondary Air Systems", Journal of Engineering for Gas Turbine and Power Vol. 112 pp 223-228.

[5] Peltier V., Dullenkopf K., Bauer H.-J., 2012, “Experimental Investigation of the Performance of different Bleed Air System Designs", Proceedings of ASME Turbo Expo, GT201268242, Copenhagen, Denmark.

[6] Peltier V., Dullenkopf K., Bauer H.-J., 2014, "Numerical Investigation of the Aerodynamic Behaviour of a Compressor Bleed Air System", Proceedings of ASME Turbo Expo, GT2014-25822, Dusseldorf, Germany.

[7] Gomes R., Schwarz C., Peitzer M., 2005 “Aerodynamic Investigations of a Compressor Bleed Air Configuration typical for Aeroengines", ISABE 2005-1264.

[8] Gomes R., Schwarz C., 2006 "Experimental Investigation of a Generic Compressor Bleed System”, ASME IGTI Turbo Expo GT2006-90458.

[9] Grimshaw S. D., Pullan G., Walker T., 2014 "Bleed Induced Distortion in Axial Compressors", ASME IGTI Turbo Expo GT2014-26195.

[10] Grimshaw S. D., Pullan G., Haynes T.P., 2015 "Modelling Non-Uniform Bleed in Axial Compressors", Proceedings of ASME Turbo Expo 2015 GT2015-43493.

[11] Aufderheide T.; Friedrichs J.; Becker B., 2018 "Effects of Secondary Air Removal on the Aerodynamic Behaviour of a Compressor Cascade Flow", Global Power and Propulsion Society, Zurich, Switzerland.

[12] Achmus M., Friedrichs J., Becker B., 2019, "Experimental Investigation of the Flow Characteristics in a Circumferential Bleed Port Geometry and the Interaction with a 1.5stage Axial Compressor", Proceedings of International Gas Turbine Conference, Paper No. TuPM16.3, Tokyo, Japan. 